\title{
Improving Mobile Robot Robustness in Visual Servoing Application
}

\author{
Laroussi Hammouda ${ }^{1}$, Hassen Mekki ${ }^{1,2}$, Khaled Kaaniche ${ }^{1,2}$ and Mohamed \\ Chtourou $^{1}$ \\ ${ }^{1}$ CEM Laboratory, University of Sfax, Tunisia \\ ${ }^{2}$ National School of Engineering of Sousse, University of Sousse, Tunisia
}

Laroussi.hmd@gmail.com

\begin{abstract}
This paper proposes a novel method to improve mobile robot robustness with respect to kinematic modeling errors during visual servoing task. Instead of using first-order error-dynamics, as it is usually done, we use the second-order error-dynamics leading to a new control law. The main aim of this approach is to guarantee a robust visual servoing scheme. In fact, the new control law ensures the convergence of the mobile robot to its desired pose even in the presence of modeling errors. Experimental results are presented to validate our approach and to demonstrate its efficiency.
\end{abstract}

Keywords: Visual servoing, error dynamics, mobile robot, modeling errors

\section{Introduction}

Computer vision is progressively playing more important role in service robotic applications since the vision sensors provide a vast amount of information on the environment in which robots move. In fact, the movement of a robot equipped with a camera can be controlled from its visual perception using visual servoing technique. Thus, the visual servoing control refers to the use of visual data to control the motion of the robot [2].

The main goal of the visual servoing scheme is to control a robotic system using visual features acquired by a visual sensor $[1,17]$. The aim is to move the robot to a desired pose regarding to a set of visual features in the viewed scene. Indeed, the control law is designed to move a robot so that the current visual features $s$, acquired from the current pose $r$, will reach the desired features $s^{*}$ acquired from the desired pose $r^{*}$, leading to a correct realization of the task. The control principle is thus to minimize the error $\mathrm{e}=\mathrm{s}-\mathrm{s}^{*}$ where $\mathrm{s}$ is a vector containing the current values of the chosen visual information, and $\mathrm{s}^{*}$ its desired values.

The basic step in image-based visual servoing is to determine the adequate set of visual features to be extracted from the image and used in the control scheme in order to obtain an optimal behavior of the robot.

Several works on visual servoing were, usually, concerned with robot manipulators where motion constraints are generally not supposed. Nevertheless, mobile robots are susceptible to nonholonomic constraints that brought to several challenges in the visual servoing task $[20,13]$. Regarding the camera position, two types of visual servoing could be distinguished: fixed-camera (eye-on-hand) visual servoing in which the visual data is acquired from stationary camera and non fixed-camera (eye-in-hand) visual servoing during which the motion of the robot induces camera motion [1, 2]. Based on the error domain, two types of visual servoing could be defined: position based visual servoing and image based visual servoing. The first one assumes that the error is defined in 3D space, 
while in image based visual servoing the error is defined in terms of image features. Image based visual servoing is more robust than position based visual servoing with respect to uncertainties and disturbances affecting the model of the robot, as well as the calibration of the camera [14].

There are more advanced approaches including hybrid, 2.5D and partitioned visual servoing [18]. These techniques are based on the combination between image based visual servoing and position based visual servoing methods [2]. For example, 2.5D visual servoing does not require a 3D model of the object and ensures a decoupled control law, but it is more sensitive to image noise [2]. This approach has been used to perform visual servoing tasks using unicycle mobile robots [19].Recent works on visual servoing are based on known robot-camera model. Therefore, the relation between the robot kinematics and the visual features variation is obtained [2, 7]. Apart from these approaches, others works are based on an estimated robot-camera model [8].

Several approaches use uncalibrated visual servoing that deal with unknown robot kinematics and unknown camera parameters [6]. An efficient approach to formulate the visual servoing as a nonlinear problem solved by a quasi-Newton method using Broyden Jacobian estimation proposed in [3]. In [4], multiple camera model-based 3-D visual servoing has used similar techniques. A novel approach of uncalibrated eye-in-hand visual servo control based on inverse fuzzy modeling, for mobile robots is proposed in [7].

Wheeled robotic platforms are typically modeled as either unicycle-type robots or carlike robots. Unicycle-type robots have two drive wheels, on the same axis, which can be driven independently, while car-like robots are typically rear wheel drive with a front wheel steering assembly.

Numerous research studies focused on the control of mobile robots $[9,10,11,15]$. Thus, highly nonlinear control techniques were developed since these systems are associated with nonholonomy constraints [21]. In the literature, the control techniques are designed mainly for unicycle-type and car-like mobile robots. Nevertheless, the control of mobile robots is related with problems that have been well studied in the literature. The last decade have seen active research in the field of nonholonomic mobile robots control. Thus, several effective control strategies are used for nonholonomic platforms [5, 15, 11].

In this work, the control of a unicycle mobile platform using a single camera attached to the robot (eye-in-hand) is addressed. During a visual servoing application and using wheeled mobile robot, many types of errors lead to undesired robot behavior.

The contribution of this paper consists in the definition of a new approach able to ameliorate mobile robot robustness regarding to kinematic modeling errors during visual servoing task. In fact, the error-dynamics considered in all visual servoing schemes were, usually, a first-order dynamics. In this paper, we propose new way to achieve visual servoing tasks based on a second-order error-dynamics.

The paper is organized as follows: Section 2 illustrates the visual servoing problem. Section 3 presents its application to nonholonomic mobile robot. We describe in section 4 our new method ensuring amelioration of control law robusteness in visual servoing task. Finally, experimental results are presented in section 5.

\section{The Visual Servoing Problem Formulation}

The visual servoing is based on the relationship between the camera motion and the consequent changes on the visual features. This relationship is expressed by the well known equation:

$$
\dot{\mathrm{s}}=\mathrm{L}_{\mathrm{s}} \mathrm{V}
$$


$\mathrm{L}_{\mathrm{s}}$ is the interaction matrix that links the time variation of $\mathrm{s}$ to the camera instantaneousvelocity $\mathrm{V}$.

The main characteristic of an image-based visual servoing task consists in determining an appropriate error function that converges to zero during the task achievement (Fig.1).

This error (e) is typically defined by:

$$
\mathrm{e}(\mathrm{t})=\mathrm{s}-\mathrm{s}^{*}
$$

where $s$ are a set of current image features and $s^{*}$ contains the desired values of the features.

The time variation of this error is given by:

$$
\dot{\mathrm{e}}=\dot{\mathrm{s}}-\mathrm{s}^{*} * \dot{\mathrm{s}}
$$

Using (1) and (3) it is possible to obtain the relationship between the camera velocity and the time variation of the error as:

$$
\dot{e}=L_{S} V
$$

Where $L_{s}$ is the interaction matrix (image Jacobian), defined as:

$$
L_{S}=\left[\begin{array}{cccccc}
-\frac{1}{z} & 0 & \frac{x}{z} & x y & -\left(1+x^{2}\right) & y \\
0 & -\frac{1}{z} & \frac{y}{z} & 1+y^{2} & -x y & -x
\end{array}\right]
$$

$\mathrm{x}$ and $\mathrm{y}$ are the metric coordinates of the features in the $2 \mathrm{D}$ image space and $\mathrm{Z}$ is the depth between the camera and the object.

An exponential decrease of the features error during the visual servoing scheme is defined as:

$$
\dot{e}=-\lambda . e
$$

Using (4) and (6) the control law used in the visual servoing context is then:

$$
V=-\lambda \cdot L_{s}^{+} e
$$

Where $\mathrm{L}_{\mathrm{s}}{ }^{+}$is the pseudo inverse of the interaction matrix $\mathrm{L}_{\mathrm{s}}$, and $\lambda$ is the control gain.

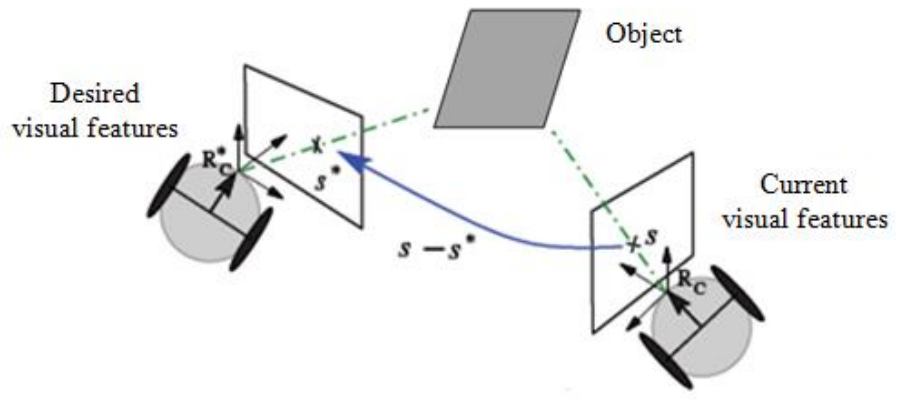

Figure 1 Visual Features Acquisition During Visual Servoing Scheme

\section{Adaptation of Visual Servoing to Nonholonomic Mobile Robot}


Generally, the visual servoing is performed with a manipulator robot. In our work, we adapt the visual servoing approach to control a unicycle-type wheeled robot with an embedded camera. The relationship between the image features velocities and the wheels velocities is a required element in the visual servoing scheme.

The kinematic model of a unicycle-type $\left(\dot{\mathrm{X}}_{\text {uni }}\right)$ robot, using Newton's notation is shown in the following equations:

$$
\begin{gathered}
\dot{x}=v \cos (\theta) \\
\dot{y}=v \sin (\theta) \\
\dot{\theta}=\omega
\end{gathered}
$$

where $(\mathrm{v}, \omega)$ are the control inputs, $\mathrm{x}$ and $\mathrm{y}$ represent the mobile robot position and $\theta$ is its orientation.

Figure 2 shows the robot-camera system where $R_{b}$ is the fixed base frame, $R_{m}$ the mobile robot frame, $\mathrm{R}_{\mathrm{c}}$ the camera frame and $\psi$ the camera tilt.

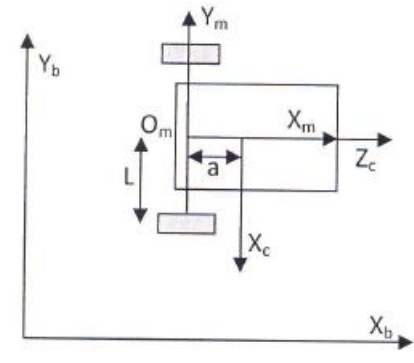

(a)

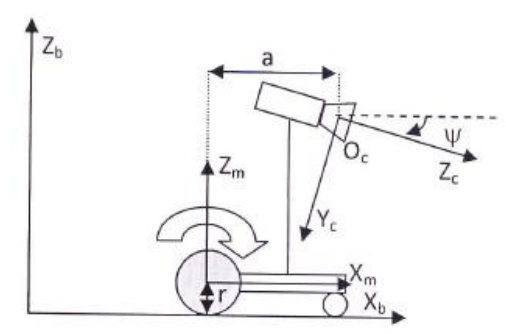

(b)

Figure 2 Robot-Camera System: (a) top view, (b) side view

Let's consider a wheeled robot with a pose defined by:

$$
\left[\begin{array}{l}
x \\
y \\
\theta
\end{array}\right]
$$

Assuming that the motion is done without sliding, the following equations describe the kinematic behavior of the robot:

$$
\begin{gathered}
\dot{x}=v_{R} \cos \theta \\
\dot{y}=v_{R} \sin \theta \\
\dot{\theta}=\omega_{R}
\end{gathered}
$$

where $\left(v_{R}, \omega_{R}\right)$ is the linear and angular velocities of the mobile robot.

We note that:

$$
V_{R}=\left[\begin{array}{c}
\frac{r}{2}\left(\varphi_{r}+\varphi_{l}\right)=v_{R} \\
0 \\
0
\end{array}\right]
$$




$$
\Omega_{R}=\left[\begin{array}{c}
0 \\
0 \\
\frac{r}{2 L}\left(\varphi_{r}-\varphi_{l}\right)=\omega_{R}
\end{array}\right]
$$

Once we have the robot inputs $\left(v_{R}, \omega_{R}\right)$, the correspondent velocities of the wheels (right and left) can be calculated according to the following equation:

$$
\left(\begin{array}{c}
\varphi_{r} \\
\varphi_{l}
\end{array}\right)=J_{m}^{-1} \cdot\left(\begin{array}{c}
v_{R} \\
\omega_{R}
\end{array}\right)
$$

where

$$
J_{m}^{-1}=\left(\begin{array}{cc}
\frac{1}{r} & \frac{L}{r} \\
\frac{1}{r} & -\frac{L}{r}
\end{array}\right)
$$

with $\mathrm{L}$ is the half distance between the robot's wheels and $\mathrm{r}$ is the radius of the wheel.

The rotation matrix $R_{m c}$ between the mobile robot frame $\left(R_{m}\right)$ and the camera frame $\left(\mathrm{R}_{\mathrm{c}}\right)$ is defined as:

$$
R_{m c}=\left(\begin{array}{ccc}
0 & -1 & 0 \\
\sin \psi & 0 & -\cos \psi \\
\cos \psi & 0 & \sin \psi
\end{array}\right)
$$

Let's note that in our case $\psi=0$.

The components of the camera kinematic screw $V c=\left[\begin{array}{c}V_{c} \\ \omega_{c}\end{array}\right]$ can be written as follows:

$$
\begin{gathered}
v_{c}=R_{m c}\left(\begin{array}{c}
v_{R} \\
0 \\
0
\end{array}\right)+R_{m c}\left(\begin{array}{c}
0 \\
a \omega_{R} \\
0
\end{array}\right)=\left(\begin{array}{c}
-a \omega_{R} \\
0 \\
v_{R}
\end{array}\right) \\
\omega_{c}=R_{m c}\left(\begin{array}{c}
0 \\
0 \\
\omega_{R}
\end{array}\right)=\left(\begin{array}{c}
0 \\
-\omega_{R} \\
0
\end{array}\right)
\end{gathered}
$$

Then,

$$
V_{c}=\left[\begin{array}{c}
-a \omega_{R} \\
0 \\
v_{R} \\
0 \\
-\omega_{R} \\
0
\end{array}\right]
$$

The relation between $\left(v_{R}, \omega_{R}\right)$ and the camera instantaneous velocity is given by:

$$
V_{c}=J_{r} \cdot\left(\begin{array}{c}
v_{R} \\
\omega_{R}
\end{array}\right)
$$


with $J_{r}=\left(\begin{array}{rr}0 & -a \\ 0 & 0 \\ 1 & 0 \\ 0 & 0 \\ 0 & -1 \\ 0 & 0\end{array}\right)$ and $a$ is the distance between the turret on which the camera is mounted and the wheels axis.

Thus, from (1) and (19) we deduce the relation between the variation of the visual features $\dot{s}$ and $\left(v_{R}, \omega_{R}\right)$ defined as:

$$
\dot{s}=J_{t} \cdot\left(\begin{array}{c}
v_{R} \\
\omega_{R}
\end{array}\right)
$$

Where

$$
J_{t}=L_{s} \cdot J_{r}=\left[\begin{array}{cc}
\frac{y}{z} & \frac{a}{z}+1+x^{2} \\
\frac{x}{z} & x y
\end{array}\right]
$$

The control law $\left(v_{R}, \omega_{R}\right)$ of the mobile robot has, then, the following expression (Fig. $3)$ :

$$
\left(\begin{array}{c}
v_{R} \\
\omega_{R}
\end{array}\right)=-\lambda \cdot J_{t}^{+} \cdot e
$$

with $J_{t}^{+}$is the pseudo inverse of the interaction matrix $J_{t}$.

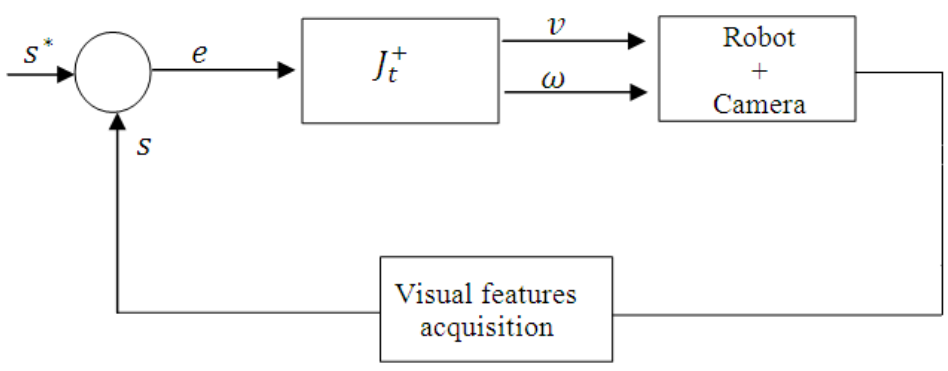

\section{Figure 3 The Visual Servoing Scheme}

\section{Visual Servoing Based on Second-order Error-Dynamics}

In the literature, the error usually used in visual servoing applications has an exponential decrease defined by:

$$
\dot{e}+\lambda e=0
$$

This error-dynamics leads to the traditional control law of equation (7).

In this work, we move from the first-order error-dynamics to the second-order errordynamics defined by the following equation:

$$
\ddot{e}+k_{1} \dot{e}+k_{2} e=0
$$


Thus,

$$
\dot{e}=-\frac{1}{k_{1}} \ddot{e}-\frac{k_{2}}{k_{1}} e
$$

The new control law, used in the visual servoing scheme, has then the following form:

$$
V=-L_{S}^{+}\left(\frac{1}{k_{1}} \ddot{e}+\frac{k_{2}}{k_{1}} e\right)
$$

Where the second derivative of the error is defined by:

$$
\ddot{e}=\frac{e_{k}-2 e_{k-1}+e_{k-2}}{T^{2}}
$$

With $e_{i}=s_{i}-s_{i}^{*}$

Then, our new control law used in the visual servoing scheme will be as follows:

$$
V=-L_{s}^{+}\left[\frac{1}{T^{2} k_{1}}\left(e_{k-2}-2 e_{k-1}\right)+\left(\frac{1}{T^{2} k_{1}}+\frac{k_{2}}{k_{1}}\right) e_{k}\right]
$$

This approach ensures more robustness regarding to mobile robot modeling errors during visual servoing task.

We choose the coefficients $\mathrm{k}_{1}$ and $\mathrm{k}_{2}$ in such a way that the roots of the characteristic polynomial of (24) have negatives real parts.

The following equations present the kinematic model of the mobile robot with error $(\rho)$.

$$
\begin{aligned}
& \dot{x}=v_{R} \cos \theta+\rho \\
& \dot{y}=v_{R} \sin \theta \\
& \dot{\theta}=\omega_{R}
\end{aligned}
$$

The kinematic modeling errors induce mobile robot singularities. Thus, during a positioning task, the robot diverges and doesn't reach its desired pose. In fact, in the presence of modeling error, the movement performed by the mobile robot doesn't correspond to the velocities generated by the control law.

Using this new error-dynamics the robot converges to its desired pose even in the presence of modeling errors. However, this convergence was not guaranteed in visual servoing scheme based on an exponential decrease of the error.

\section{Experimental Results}

The visual servoing system used here is the eye-in-hand system, in which the camera is linked to the mobile robot and facing the whole environment. In this work, the imagebased visual servoing is considered. The error signal is measured directly in the image and mapped to the robot control law.

We illustrate the results of a set of experiments conducted using a mobile robot with an embedded camera. In each case, the mobile robot is first moved to its desired pose $r^{*}$ and the corresponding image $\mathrm{I}^{*}$ is acquired. From this desired image, we extract the desired visual features $\mathrm{s}^{*}$. The robot is then moved to a random pose $r$ and the initial visual features s are extracted. The velocities $(\mathrm{v}, \omega)$ computed, at each frame, using the control law, are sent to the robot until its convergence. The interaction matrix is calculated at each 
frame of the visual servoing scheme. We conduct our experiments on a virtual platform of VRML, therefore we can recuperate, at each frame, the pose of the mobile robot in terms of position along two translational axes and around one rotational axe.

During our experiments, the visual features used are the coordinates of four points obtained using the Harris detector. We involve here our new method based on secondorder error-dynamics and we compare it to the traditional visual servoing approach based on a first-order error-dynamics.

In a first experiment, we take as initial positioning error: $\Delta r_{i n t}=\left(6 \mathrm{~m}, 3 \mathrm{~m}, 11^{\circ}\right)$. We suppose that the kinematic model of the robot doesn't include any error. The generated control law is based on the new expression of the error decrease. Figures $4 \mathrm{a}$ and $4 \mathrm{~b}$, show, respectively, the initial (s) and the desired $\left(\mathrm{s}^{*}\right)$ visual features. We illustrate on Fig. $4 \mathrm{c}$ the visual features paths during the positioning task while the decrease of the error norm is shown on Fig. 4d.

In a second experiment, the initial positioning error is $\Delta \mathrm{r}_{\text {int }}=\left(8 \mathrm{~m}, 4 \mathrm{~m}, 17^{\circ}\right)$. We suppose now the existence of error in the kinematic model of the wheeled mobile robot: $\rho=0.5$. In the presence of such a modeling error, the traditional method based on the exponential decrease of the error doesn't ensure the convergence of the robot to its desired pose.

In fact, the mobile robot gets at a local minimum (Fig. 5e). It is clear from Fig. 5e that the system has been attracted to a local minimum far away from the desired configuration. Figure 5a shows the visual features corresponding to the initial pose of the robot. Figure $5 \mathrm{~b}$ illustrates the visual features corresponding to the final pose of the robot which is, in this case, different from the desired pose. The translational and the rotational positioning errors during the positioning task (Fig. 5c and Fig. 5d) don't converge all to zero due to the local minimum. We present on Fig. 5e the mobile robot path during the visual servoing task.

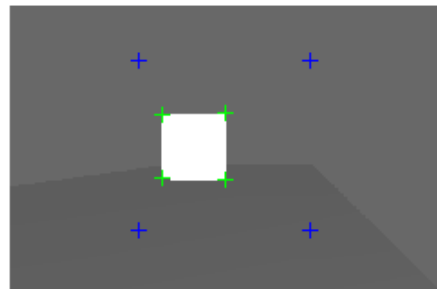

(a)

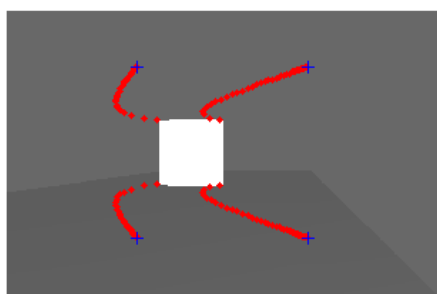

(c)

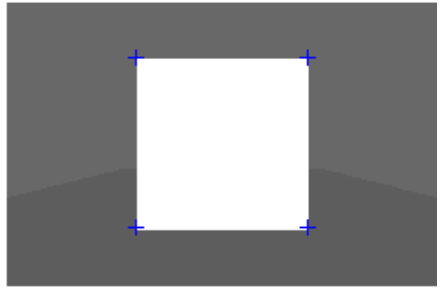

(b)

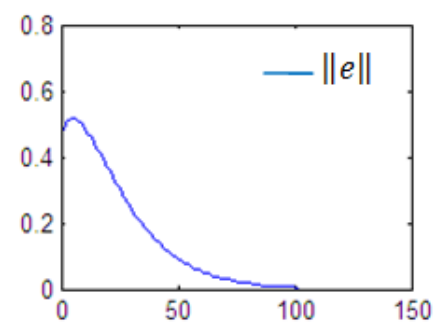

(d)

Figure 4 Visual Servoing Task: (a) Initial image, (b) Desired image, (c) Visual features paths $(d)$ error norm ( $x$ axis in frame number). 


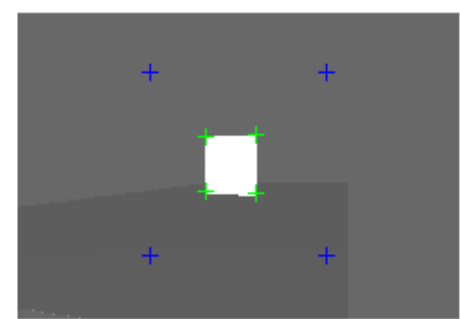

(a)

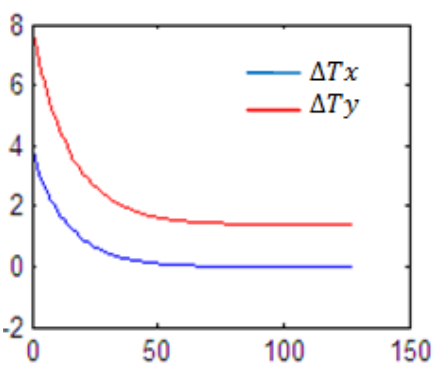

(c)

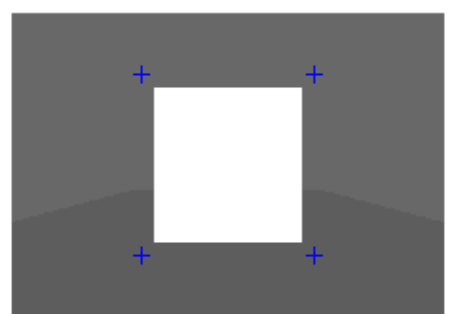

(b)

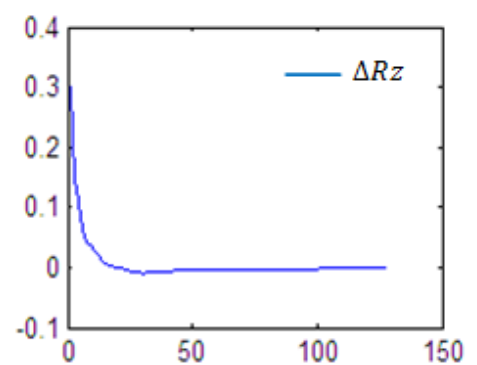

(d)

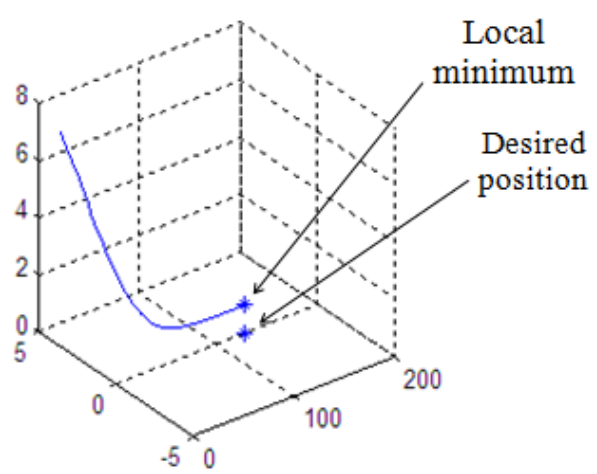

(e)

Figure 5 Visual Servoing Task ( $x$ axis in frame number) : (a) Initial image, (b) Final image, (c) Translational positioning errors: $\Delta T x$ and $\Delta T y$ in meter $(m)$, (d) Rotational positioning error: $\Delta R z$ in radian ( $\mathrm{rad})$, (e) Mobile robot path.

In a third experiment, the visual servoing task is performed using the new errordynamics with $\mathrm{k}_{1}=8$ and $\mathrm{k}_{2}=39$. We remark that the mobile robot converges to its desired pose without singularities.

Figure 6a illustrates the initial visual features while the desired ones are shown on Fig. $6 \mathrm{~b}$. Figures $6 \mathrm{c}$ and $6 \mathrm{~d}$ present, respectively, the linear $(\mathrm{v})$ and the angular $(\omega)$ velocities of the mobile robot. The translational positioning errors $(\Delta \mathrm{Tx}, \Delta \mathrm{Ty})$ between the current and the desired pose during the positioning task are shown on Fig. 6e. The rotational positioning error $(\Delta \mathrm{Rz})$ is illustrated on Fig. $6 \mathrm{f}$. We present on Fig. $6 \mathrm{~g}$ the mobile robot path during the visual servoing task. 


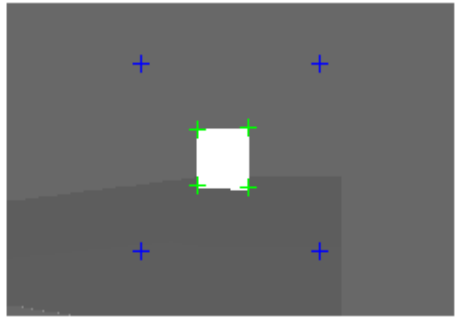

(a)

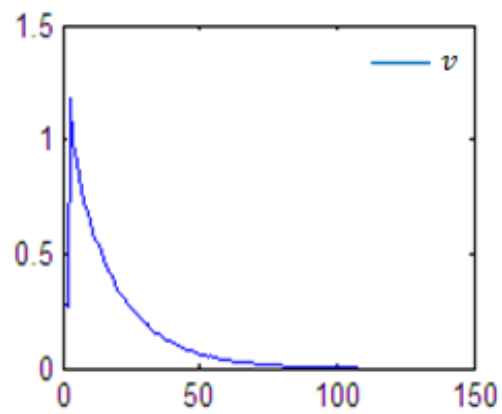

(c)

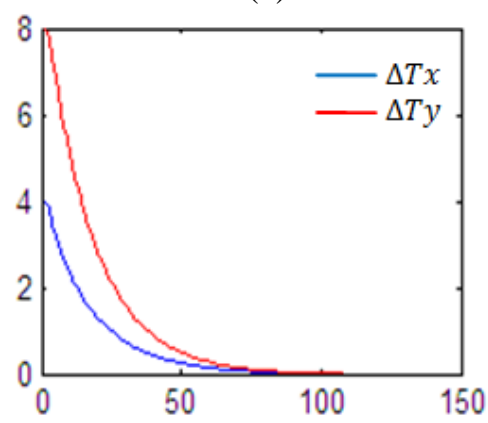

(e)

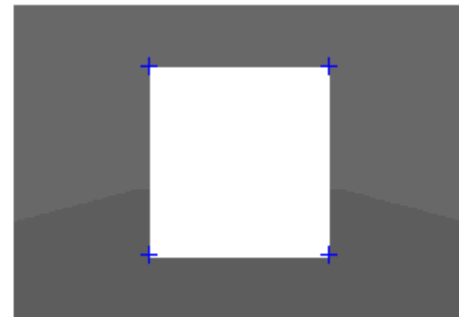

(b)

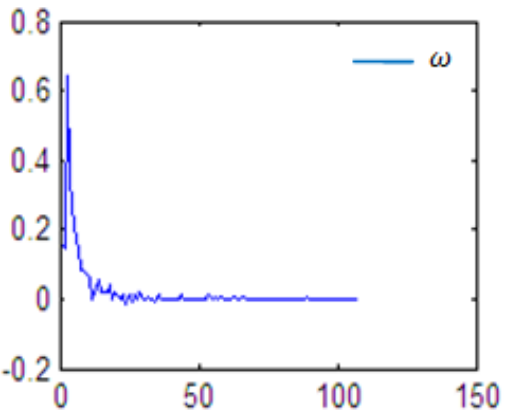

(d)

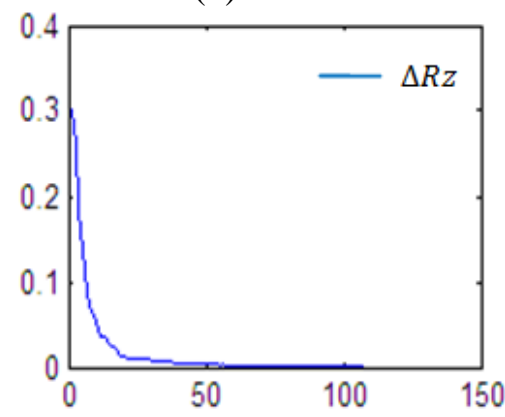

(f)

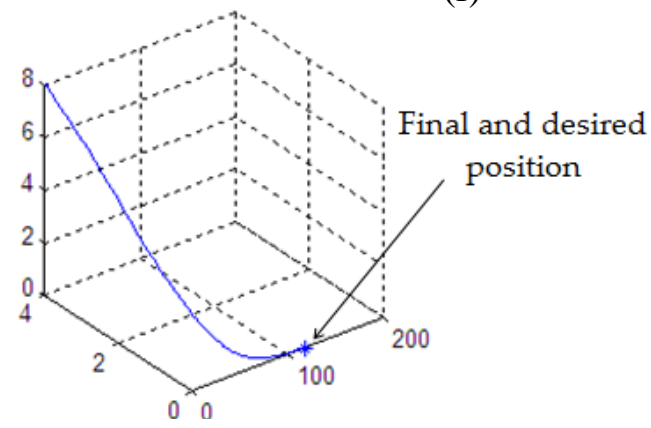

(g)

Figure 6 Visual Servoing Task ( $x$ axis in frame number) : (a) Initial image, (b) Desired image, (c) Linear mobile robot velocity in $(\mathrm{m} / \mathrm{s})$, (d) Angular mobile robot velocity in ( $\mathrm{rad} / \mathrm{s})$, (e) Translational positioning errors: $\Delta \mathrm{Tx}$ and $\Delta \mathrm{Ty}$ in meter (m), (f) Rotational positioning error: $\Delta \mathrm{Rz}$ in radian (rad), (g) Mobile robot path.

\section{Conclusion}

In this paper we focused on the application of visual servoing to nonholonomic mobile robot. Thus, we present a novel technique to achieve robust control law in visual servoing context. In the literature, the error, used in the building of the control law, was usually characterized by an exponential decrease. This work proposed new way of error decrease that guaranties robust visual servoing. We replace the first-order error-dynamics by the 
second-order error-dynamics. In such a way, the mobile robot reaches its desired pose even in the case of kinematic modeling errors.

Future works can be intended to verify the robustness of our approach with respect to partial occlusions and large illumination changes.

\section{References}

[1] F. Chaumette and S. Hutchinson, "Visual servoing and visual tracking", Handbook of Robotics, B. Siciliano, O. Khatib (eds.), Springer, chapter 24, (2008), pp. 563-583.

[2] F. Chaumette and S. Hutchinson, "Visual servo control, Part I: Basic approaches", IEEE Robotics and Automation Magazine, vol. 13, no. 4, (2006), pp. 82-90.

[3] S. Hutchinson, G. D. Hager and P. I. Corke, "A tutorial on visual servo control”, IEEE Transactions on Robotics and Automation, vol. 12, no. 5, (1996), pp. 651-670.

[4] E. Malis, F. Chaumette and S. Boudet, "2 1/2 D Visual Servoing", IEEE Transactions on Robotics and Automation, vol. 15, no. 2, (1999), pp. 238-250.

[5] M. Jagersand and R. Nelson, "On-line Estimation of Visual-Motor Models using Active Vision", Proceedings ARPA Image Understanding Workshop, (1996).

[6] J. Stavitzky and D. Capson. "Multiple Camera Model Based Visual Servo". IEEE Trans. On Robotics and Automation, vol. 16, no.6, (2000), pp. 732-739.

[7] P. Morin and C. Samson, "Motion Control of Wheeled Mobile Robots", Springer Handbook of Robotics, chap. 34, (2008), pp. 799-825.

[8] W. E. Dixon, D. M. Dawson, E. Zergeroglu and A. Behal, "Adaptive tracking control Of a wheeled mobile robot via an uncalibrated camera system", IEEE Transactions on systems, man, vol. 31, no. 3, (2001), pp. 341-352.

[9] P. Goncalves, P. Lopez, P. Torres and J. Sequeira, "Fuzzy Visual servo Control applied to Autonomous Driving”, EPIA'2011, (2011), pp. 440-452.

[10] P. Goncalves, L. Mendon, J. Sousa and J. Pinto, "Uncalibrated eye-to-hand visual servoing using inverse fuzzy models", IEEE transactions on fuzzy systems, vol. 16, no. 2, (2008), pp. 341-353.

[11] G. Artus, P. Morin and C. Samson, "Tracking of an omnidirectional target with a nonholonomic mobile robot", Proceeding of IEEE Conf. on Advanced Robotics (ICAR), (2003), pp. 1468-1473.

[12] M. Maya-Mendez, P. Morin and C. Samson, "Control of a nonholonomic mobile robot via sensorbased target tracking and pose estimation", Proceedings of IEEE/RSJ Int. Conf. Intell. Robots System, (2006), pp. 5612- 5618.

[13] W. Dixon, D. Dawson, E. Zergeroglu and F. Zhang, "Robust tracking and regulation control for mobile robots", International Journal of Robust and Nonlinear Control, vol.10, (2000), pp. 199-216.

[14] P. Morin and C. Samson, "Trajectory tracking for nonholonomic vehicles: overview and case study", Proceedings of 4th Inter. Workshop on Robot Motion Control, editor K. Kozlowski, (2004), pp. 139153.

[15] M. Fruchard, P. Morin and C. Samson, "A framework for the control of nonholonomic mobile manipulators”, RoMoCo'04. International Journal of Robotics Research, vol. 25, no. 8, (2006), pp. 745-780.

[16] G. L. Mariottini, G. Oriolo and D. Prattichizzo, "Image-Based Visual Servoing for Nonholonomic Mobile Robots Using Epipolar Geometry”, IEEE Transactions on Robotics, vol. 23, no. 1, (2007), pp. 87-100.

[17] W. Dong and W. Xu, “Adaptive tracking control of uncertain nonholonomic Dynamic system”, IEEE Transactions on Automatic Control, vol. 46, no. 3, (2001), pp. 450-454.

[18] R. F. Das, R. Kumar, J. Ostrowski, J. Spletzer and C. Taylor, "A vision based formation control framework", IEEE Transactions on Robotics and Automation, vol. 18, no. 5, (2002), pp. 813-825.

[19] F. Chaumette and S. Hutchinson, "Visual servo control, part II: Advanced approaches", IEEE Robotics and Automation Magazine, vol. 14, no. 1, (2007), pp. 109-118.

[20] E. Malis and F. Chaumette, "2-1/2-D visual servoing with respect to unknown objects through a new estimation scheme of camera displacement”, Int. J. Comput. Vis., vol. 37, no. 1, (2000), pp. 79-97.

[21] Y. Fang, W. E. Dixon, D. M. Dawson and P. Chawda, "Homography based visual servo regulation of mobile robots", IEEE Trans. Syst., Man, Cybern. B: Cybern, vol. 35, no. 5, (2005), pp. 1041-1050.

[22] X. Zhang, Y. Fang and X. Liu, "Visual Servoing of Nonholonomic Mobile Robots Based on a New Motion Estimation Technique", Joint 48th IEEE Conference on Decision and Control and 28th Chinese Control Conference, Shanghai, P.R. China, (2009) December 16-18. 
[23] G. Mariottini, D. Prattichizzo and G. Oriolo, "Image-based Visual Servoing for Nonholonomic Mobile Robots with Central Catadioptric Camera", Robotics and Automation, ICRA '06. Proceeding 2006 IEEE International Conference, (2006), pp. 538 - 544.

Authors

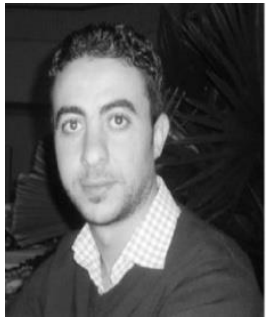

\section{Laroussi Hammouda}

He received his engineering diploma in electrical engineering from the National Engineering School of Sousse-Tunisia in 2008, his Masters degree from same Engineering School in 2009. He is currently a Ph.D student in the CEM Laboratory in the National Engineering School of Sfax-Tunisia. His current research interests include computer vision. visual servoing. Perception for

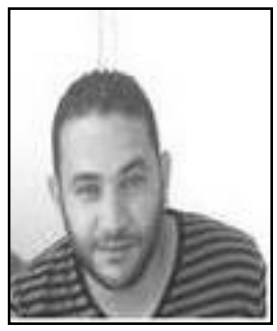

\section{Khaled Kaaniche}

He received his engineering diploma in electrical engineering from the National Engineering School of Sfax-Tunisia in 2000, his $\mathrm{Ph} . \mathrm{D}$ degree in electrical engineering from UPJV, Amiens, France in 2005. He is currently the director of computer engineering department of the National Engineering School of Sousse-Tunisia. His current research interests include perception for robotics, visual servoing, image Processing and Pattern Recognition.

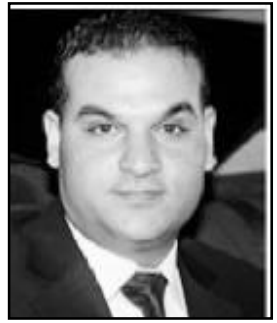

\section{Hassen Mekki}

He received his BS and MS in electrical engineering from the National Engineering School of Tunis-Tunisia, in 1999 and 2000, respectively. He received his Ph.D degree and the research habilitation from the National Engineering School of SfaxTunisia, in 2005 and 2013, respectively. He is currently a professor in the National Engineering School of Sousse. His recearch intereste include adantive intellioent rontrol and

\section{Mohamed Chtourou}

He received his engineering diploma in electrical engineering from the National Engineering School of Sfax-Tunisia in 1989, his Ph.D degree from the National Polytechnic Institute of Toulouse in 1993 and his research habilitation from the National Engineering School of Sfax-Tunisia in 2002. He is currently a professor in the National Engineering School of Sfax-Tunisia. His current research interests include learning algorithms, artificial neural networks and their engineering application fuzzy system and intelligent control. 